\title{
8. Identity Constructions in Luxembourg
}

\author{
Rachel Reckinger, Christian Wille, Christian Schulz
}

The focus of this book was on constructions of identities in Luxembourg. This deliberately 'comprehensive' wording indicates that it was not national identity(ies) or cultural identity(ies) that we were primarily concerned with but rather processes of the subject-related and actor-related everyday "Identitätsarbeit" (Keupp et al. 2006) ${ }^{2}$ - something we have conceptualised with the term Doing Identity. Against this background, four particularly relevant subject areas were examined in more detail: the linguistic and everyday-cultural practices, which occur more on the negotiational level, on the one hand, as well as the space-related constructions and confrontations between images of self and others, which are to be found more at the representational level, on the other. These four subject areas, which respectively focus on languages, everyday cultures, spaces and images, were systematically linked to the cross-cutting theme of social structure analysis by milieus (Vester et al. 2001). It was our particular concern to document the dynamic and dialogical character of identity options and constitutions. With varying degrees of emphasis, the respective chapters consequently take politically and medially desired or projected attributions of identities (top-down identifications of) into account as well as appropriations of identities (bottom-up identifications with).

This conceptual approach from the perspective of Doing Identity always refers to the dimension of the development and changeability of identities. Taking linguistic identities as an example, it becomes abundantly clear that equating language usage and identities would produce a foreshortened view. In fact, the study we conducted on the practised linguistic consensus and the publicly negotiated positions on language provides insights into identity constructions which cannot be defined by a simple formula. They partly reflect divergent relationships between appropriated and attributed identities, different practices of multilingualism and a wide range

1 | Personal translation: "Identity work".

2| See section 2.1. 
of ideas concerning 'good' and 'bad' multilingualism. The authors finally arrive at the conclusion that an increased awareness and social acceptance of the various linguistic standards and multilingual constellations that were identified in the course of this study can make an essential contribution to social cohesion in Luxembourg. Furthermore, using linguistic and visual images, we explored the symbolic representations of identities. The areas of communication investigated for this purpose, functioned, in the context of selected media events, as vectors for 'imagined communities' and for various constructions of Luxembourg, which are based on a rich repository of collective symbols. Social change and plurality have produced both continuities and discontinuities in their constitutional mechanisms and functionalities, which express themselves medially. The relationship between attributed and appropriated identities, which can be described as multi-faceted, is determined in Luxembourg by this medially-based application. Furthermore, under the keyword 'geographies', the authors established various space-related constructions of Luxembourg and, at different scale levels, scrutinised the identity characteristics that revealed themselves in the process. First of all, we examined, as crystallisation points of spatial identities, the representations and semiotic strategies articulated in the tourist discourses. It appeared that the identity constructions within the spatial levels we examined are partially very heterogenic, yet that they always indicate - also from a diachronic perspective - a certain uniqueness, exceptionality and in particular a diversity, which mesh with the notion of a 'Luxembourg entity' in a contrasting and ambivalent manner. In view of the idealising character of the public tourist discourse on the one hand and the representations and social practices of Luxembourg's resident population on the other, the relationship between appropriated and attributed identities, as elucidated in this study, can only be described as divergent. Such divergences as well as ambivalences of identity constructions were also found in different areas of everyday culture. With respect to the relationship between appropriated and attributed identities in the context of different notions of 'good' food, we were able to establish an idealising typology (adaptation, opposition, autonomy). This reflects contradictory forms of 'everyday logic' as well as the living and experiencing of gender roles or the perception of cross-border workers, which each pragmatically break open the binary 'either-or logic' and only become accessible from a perspective of 'as well as'.

The research findings, whose main points we have highlighted above, clearly indicate the nature of identities: the identifications and identities empirically investigated in Luxembourg are as varied as they are complex. This means that concise and therefore homogenising, static and foreshortened equations - in particular concerning collective identity(ies) - can never be anything but normative and ideological. Therefore, we will forego generalising summaries and refer to the constitutive ambivalences of identity constructions discovered in the course of our survey. Appropriated and attributed identity patterns generally exhibit more or less distinctive discrepancies, which emanate from the interaction between these 
categories, respectively from their communicative relationship. Furthermore, the respective identification options or identification practices as such are alterable, plural and polysemic (and therefore potentially contradictory). In this respect, identities - as permanent but always temporary resultants of the aforementioned communicative processes - always comprise challenges and potential for change. On account of the socio-cultural milieu affiliations and the respective resources of the subjects, this can however "only' mean a "Veränderung in Grenzen"3 (Straub 2004: 284).

These dynamics can be sympathetic (e.g. in the case of tourism discourses or advertising), critical (e.g. in the case of gender constructions, food standards and eating habits, artistic or journalistic critique) or conflictual (e.g. in the case of the crossborder worker issue or multilingualism). The most significant potential for conflict can be found in those aspects of social life, which are metonymically abstracted and can be used for political-social purposes as collective symbols, capable of consensus because they unite polysemic, i.e. different, perspectives. For this reason, the issue of language became particularly topical. It seems to condense the social change in Luxembourg and the fears coupled to it, although Luxembourgish is currently spoken by more people than ever before. Therefore, in particular for those areas with a high potential for conflict, the following statement applies: "Identität wird nur in ihrer Krise zum Problem" 4 (Eickelpasch/Rademacher 2004: 5), since in these instances it is always more a matter of subjective perceptions, feelings of belonging, self-assessments, and less one of objective 'facts'. Still, the 'working out' of identities on the part of the subjects should be seen as a performance of continuity and on no account as something substantially adherent to their selves. This self-relationship is evident in synchronous as well as in diachronic ways of experiencing society and the world and is narratively processed, communicated and passed on. This process of Doing Identity aims at a "flexible Kontinuität und Sinnhaftigkeit des Selbst" 5 and serves less for the depiction or definition of objective 'facts' (Straub 2004: 284-286).

The results of the three-year long interdisciplinary research work ${ }^{6}$ presented in this volume reflect the wide range of identity options in Luxembourg and also the pluralism and changeability of identification practices. Since categories that

3 | Personal translation: "Change within limits".

4 | Personal translation: "Only when in a crisis does identity become a problem".

5 | Personal translation: "Flexible continuity and meaningfulness of the self".

6 | The project benefited to a very high degree from the comprehensive sharing of information among researchers and scientists of different disciplines as well as from the interactively structured scientific culture within the IPSE research unit (Identités, Politiques, Sociétés, Espaces) at the Faculty of Language and Literature, Humanities, Arts and Education (FLSHASE) of the University of Luxembourg. A subsequent project, currently in preparation, will also be embedded in this context. It will deal with the transnational dimension of identity constructions. 
are perceived as traditional and providing orientation are increasingly losing their meaning, issues of social cohesion in Luxembourg and beyond can no longer align themselves with a notion of societies as normatively integrated and homogeneous groups. Rather, it is a matter of opening up to and acknowledging social pluralism and internal differentiation.

From the perspective of a critical accompanying research of current social developments, questions of social cohesion must therefore aim at the make-up and dynamism of social interactions and on the meshing of different identity concepts in cultural practice. With the findings of the research project "IDENT Socio-Cultural Identities and Identity Policies in Luxembourg" presented here, the authors hope to have contributed in some degree to injecting the current intensive political, social and medial debate around identity questions in Luxembourg and elsewhere with a hightened awareness for more differentiating considerations and less reductionist argumentation patterns that regard social diversity and multiple identity patterns as a 'standard scenario' rather than as a 'problem'. 


\section{Aus:}

\section{IPSE (ed.): Doing Identity in Luxembourg. Subjective Appropriations - Institutional Attributions - Socio-Cultural Milieus. Bielefeld, transcript, 2011.}

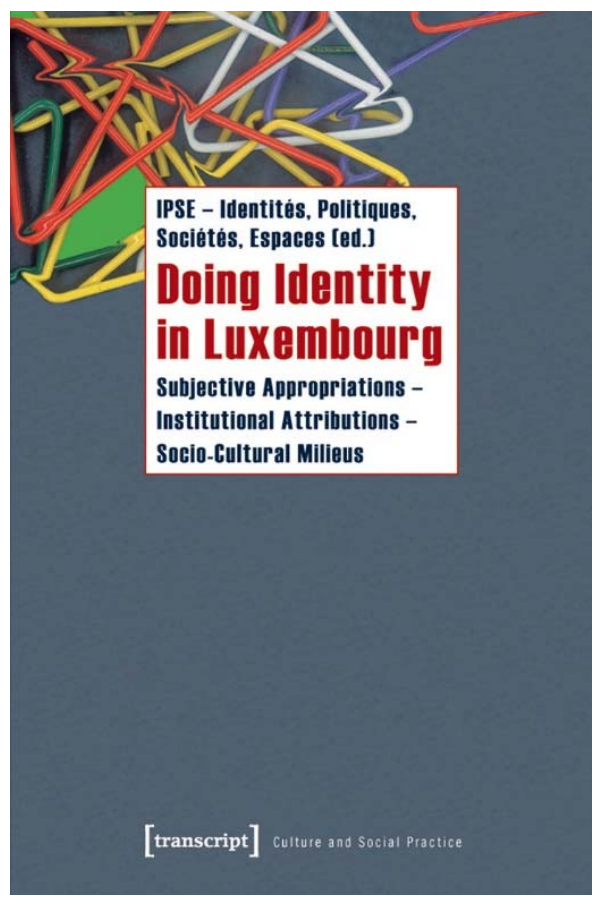

This empirical study provides insights about a society that has hitherto largely eluded scientific investigation and observes the processes of identity construction in globalised conditions. The interdisciplinary team of authors exposes the processes of subjective appropriations and institutional attributions at work in the fields of languages, spaces, perceptions of self and others as well as everyday cultures, and identifies for the first time socio-cultural milieus in the Grand Duchy. The findings of the three-year research project uncover the ambivalences and dynamics of a multicultural and multilingual society. 


\section{Content}

I. Preface $\mid 7$

Rachel Reckinger, Christian Schulz, Christian Wille

2. Researching Identity Constructions $\mid 11$

Rachel Reckinger, Christian Wille

2.1 A theoretical-conceptional approach to identities $\mid 11$

2.2 Methodology and Interdisciplinary Collaboration | 24

2.3 References | 35

3. Socio-Cultural Milieus in Luxembourg | 39

Wilhelm Amann, Fernand Fehlen, Georg Mein

3.1 Changing Social Structures. From the vertically orientated concept of stratification to the horizontally diversified milieu landscape 39

3.2 Profiles of Socio-Cultural Milieus in Luxemburg | 50

3.3 Final remark $\mid 62$

3.3 References $\mid 62$

4. Languages and Identities $\mid 6_{5}$

Peter Gilles, Sebastian Sefla, Heinz Sieburg, Melanie Wagner

4.1 Introduction: Germanic/Romance Diglossia | 65

4.2 Key Survey Results Concerning the Linguistic Situation | 68

4.3 Controversies in Readers' Letters concerning the Linguistic Situation | 81

4.4 'Linguistic Landscape': Public Signage and Multilingualism | 93

4.5 Conclusion: Linguistic Polynomia as an Opportunity $\mid 103$

4.6 References $\mid 104$

5. Spaces and Identities $\mid 107$

Marion Colas-Blaise, Sylvie Freyermuth, Sonja Kmec,

Gian Maria Tore, Christian Schulz

5.1 Introduction: Geographic Discourses and Tourism Practice | 107

5.2 Between Seduction and Reality: Luxembourg City and its Discourses $\mid 110$

5.3 Representing and Revaluating the City: the Geo-Graphy and Visual Identity of Luxembourg City $\mid 120$ 
5.4 Between National Unity and Regional Individualism 'Sways the Heart of Luxembourg' | 125

5.5 The Image of Luxembourg as a 'Melting Pot' | 133

5.6 The "Greater Region" - Spatial Constructions between Desire and Reality | 144

5.7 Conclusion: Spatial Identities - Multiple Readings?| 155

5.8 References 158

6. Images and Identities $\mid 165$

Wilhelm Amann, Viviane Bourg, Paul Dell, Fabienne Lentz, Paul Di Felice, Sebastian Reddeker

6.1 Images of Nations as 'Interdiscourses'. Preliminary Theoretical Reflections on the Relation of 'Images and Identities': The Case of Luxembourg | 165

6.2 Symbolisms in the Media-Based Perceptions of Self and Others of Luxembourg $\mid 168$

6.3 The Superjhemp Saga - an Ideal Projection Screen 179

6.4 Collective Symbols and (New) Identity Options in Luxembourg's Advertising $\mid 190$

6.5 Decontextualising and Deconstructing Representations of Identity: an Analysis of the Works of Seven Photographers | 202

6.6 The Representation of Italian Immigrants in the Exhibition Catalogue Retour de Babel | 216

6.7 Conclusions: National Identities in a Post-national Age?|225

6.8 References $\mid 226$

\section{Everyday Cultures and Identities $\mid 233$}

Christel Baltes-Löhr, Agnes Prüm, Rachel Reckinger, Christian Wille

7.1 Introduction: On the Reciprocal Relevance of Everyday Cultures and Identity Constructions | 233

7.2 Permanent Performances: Gender in Motion | 238

7.3 'Good' Food. Oscillation between Political Concept and Individual Everyday Practice | 255

7.4 Cross-Border Workers as Familiar Strangers | 271

7.5 Conclusions: Identities and Ambivalences of Everyday Cultures $\mid 283$

7.6 References $\mid 285$

8. Identity Constructions in Luxembourg | 291

Rachel Reckinger, Christian Wille, Christian Schulz

Authors | 295 